\title{
Angiogenesis in the brain after sevoflurane anesthesia in rats
}

\author{
Takashi Horiguchi MD',Yoko Masaki PhD', Koichi Kawamura PhD², Toshiaki Nishikawa MD' \\ I Department of Anesthesia and Intensive Care Medicine, Akita University Graduate School of Medicine,Akita, JAPAN \\ ${ }^{2}$ Research Institute for Science and Engineering, Waseda University, Tokyo, Japan
}

\section{Background and Goal of study}

- Although degeneration of brain cells including apoptosis has been demonstrated after inhalation of volatile anaesthesics in many reports, there is little information about the restoration process of the damaged cells after inhaled anesthesia.

- To approach the restoration process in the brain cells, we compared the ultrastructural changes in the brain cells just after sevoflurane anesthesia and those 7 days after sevoflurane anesthesia in rats.

\section{Material and Methods}

- We divided SD rats into three groups: the group just after sevoflurane $(n=3)$, the group 7 days after sevoflurane $(n=3)$ and the control group $(n=3)$.

- Spontaneously breathing rats were anesthetized with $2 \%$ sevoflurane, $33 \%$ oxygen in nitrogen for $2 \mathrm{~h}$ in the group just after sevoflurane.

- Just after the end of anesthesia, the chest was opened through a median sternotomy under intraperitoneal (IP) pentobarbital anesthesia $(100 \mathrm{mg} / \mathrm{kg})$.

- A catheter was inserted through the apex of the left cardiac ventricle and advanced into the ascending aorta for infusion of $0.1 \mathrm{M}$ cacodylate buffer ( $\mathrm{pH}$ 7.4).

- The right atrium was inserted to drain the perfusion fluid. These were postfixed in $2 \%$ glutaraldehyde solution in 0.1 $M$ cacodylate buffer ( $\mathrm{pH}$ 7.4).

- The brain was removed and embedded in Epon, and ultrathin sections were made.

- These were stained with lead citrate and uranyl acetate and were observed in a transmission electron microscope (H-7650, HITACHI, Tokyo, Japan).

- The group seven days after sevoflurane and the control group were anesthetized with IP pentobarbital.

- The other treatments were the same as the group just after sevoflurane.

\section{Results and Discussion}

- In both of the sevoflurane groups, there were many degeneration cells with atrophic nuclei in both cerebral cortex and hippocampus.

- In the group 7 days after sevoflurane, many capillaries of a few micro-meter in diameter were found.

- Its endothelial cells had large nuclei, various rich organelle and small vascular cavity.

- The characteristics of these vessels were immature blood vessel (I).
- These vessels described above were not found in the just after sevoflurane and the control groups.

- These findings suggest that angiogenesis is induced in the restoration process of the degenerated brain cells after sevoflurane inhalation.

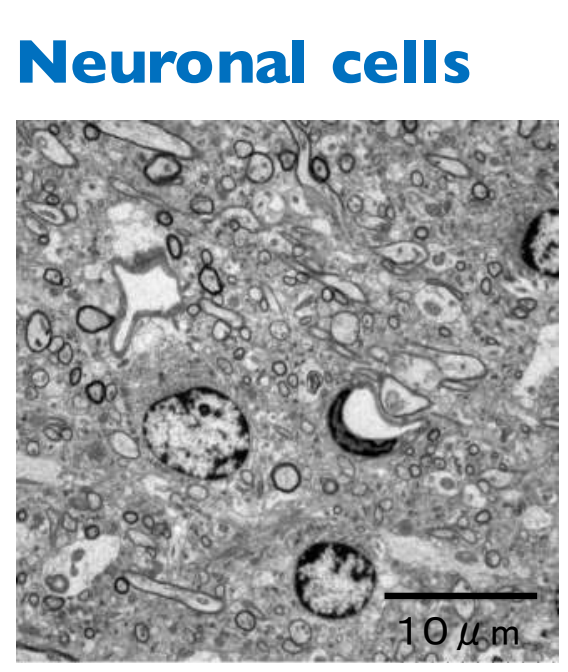

Control

(Crebral cortex)

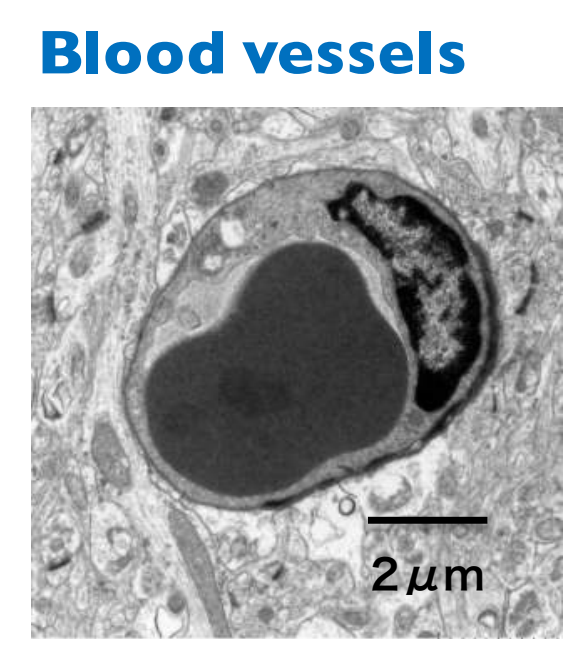

Control

(Cerebral cortex)

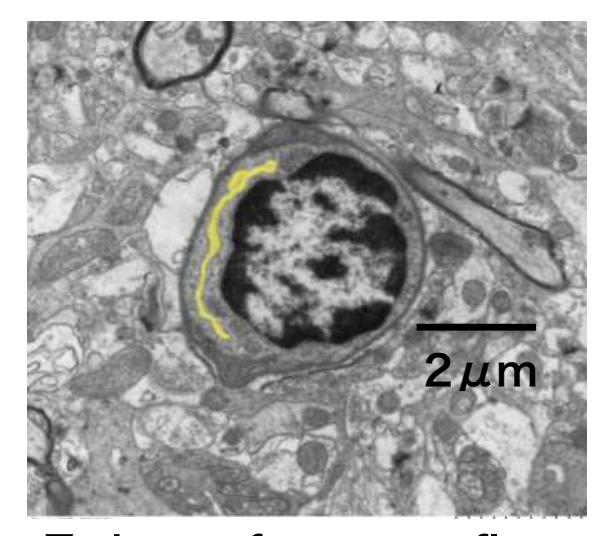

7 days after sevoflurane Immature blood vessel (Cerebral cortex)

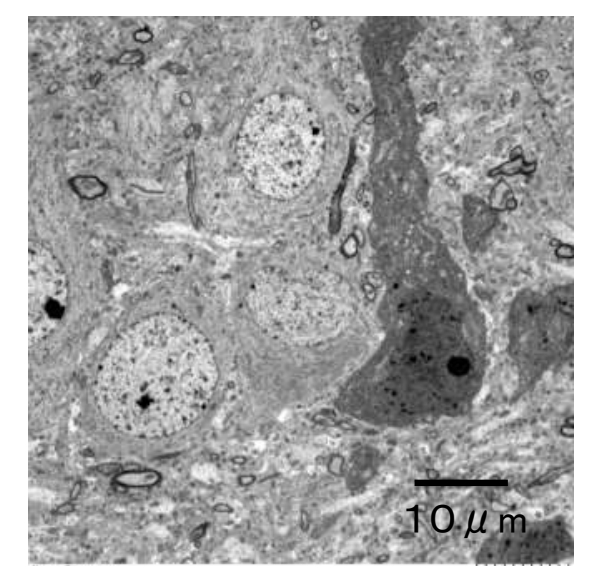

Just after sevoflurane 7 days after sevoflurane Degeneration cells (Hippocampus)

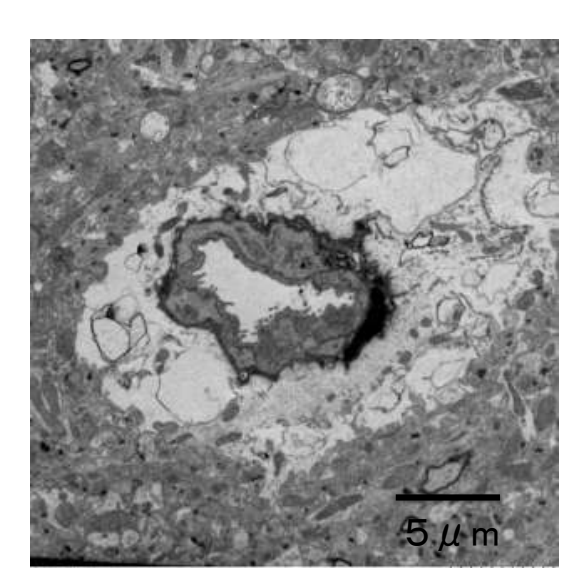

Just after sevoflurane (Cerebral cortex) Degenerated perivascular cells

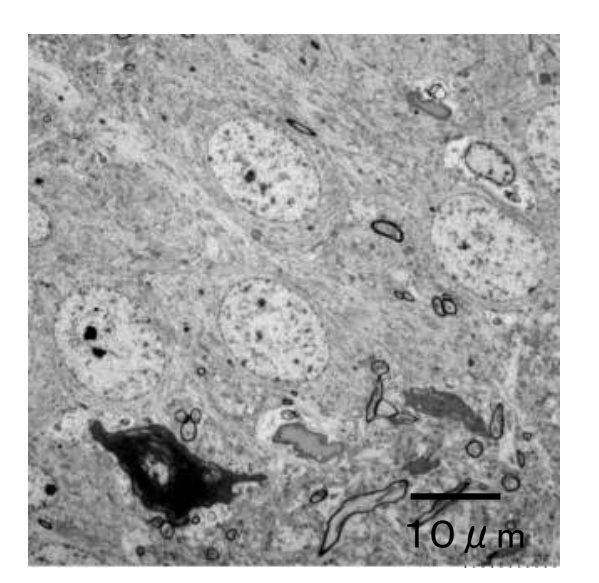

Degeneration cell (Hippocampus)

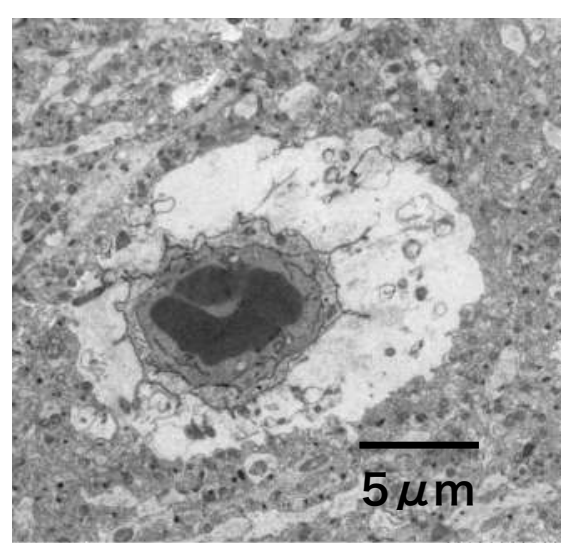

7 days after sevoflurane Degenerated perivascular cells (Hippocampus)

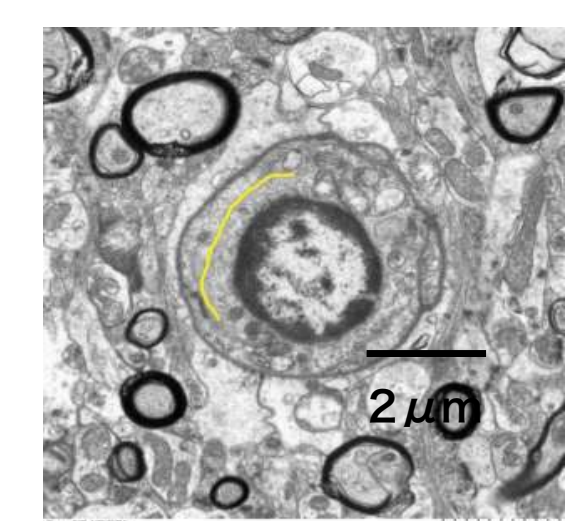

7 days after sevoflurane Immature blood vessel (Hippocampus)

Yellow parts are vascular cavity.

\section{Conclusions}

- We found many degeneration cells with atrophic nuclei and possible angiogenesis 7 days after sevoflurane anesthesia in the rat brain.

- Angiogenesis is likely to be induced in the restoration process of the degenerated brain cells caused by sevoflurane anesthesia.

\section{Reference}

(I) Hannah RS, et al.Anat Rec I78:691-710, 1974.

This work was supported by Grants in Aid for Scientific Research from Japan Society for the Promotion of Science (No. 24592292-00).

Address correspondence to Takashi Horiguchi MD

Department of Anesthesia and Intensive Care Medicine,Akita Univ. Hospital, Hondo I-I-I,Akita City,Akita 0 I0-8543, Japan

E-mail: thorigu@doc.med.akita-u.ac.jp 